\title{
Utilizing network pharmacology to explore the underlying mechanism of Radix Salviae in diabetic retinopathy
}

Chun-Li Piao ${ }^{1 *}{ }^{+}$, Jin-Li Luo $^{1 \dagger}$, De Jin ${ }^{2 \dagger}$, Cheng Tang ${ }^{1}$, Li Wang ${ }^{1}$, Feng-Mei Lian²* and Xiao-Lin Tong ${ }^{2 *}$

\begin{abstract}
Introduction: Radix Salviae (Dan-shen in pinyin), a classic Chinese herb, has been extensively used to treat diabetic retinopathy in clinical practice in China for many years. However, the pharmacological mechanisms of Radix Salviae remain vague. The aim of this study was to decrypt the underlying mechanisms of Radix Salviae in the treatment of diabetic retinopathy using a systems pharmacology approach.
\end{abstract}

Methods: A network pharmacology-based strategy was proposed to elucidate the underlying multi-component, multi-target, and multi-pathway mode of action of Radix Salviae against diabetic retinopathy. First, we collected putative targets of Radix Salviae based on the Traditional Chinese Medicine System Pharmacology database and a network of the interactions among the putative targets of Radix Salviae and known therapeutic targets of diabetic retinopathy was built. Then, two topological parameters, "degree" and "closeness certainty" were calculated to identify the major targets in the network. Furthermore, the major hubs were imported to the Database for Annotation, Visualization and Integrated Discovery to perform a pathway enrichment analysis.

Results: A total of 130 nodes, including 18 putative targets of Radix Salviae, were observed to be major hubs in terms of topological importance. The results of pathway enrichment analysis indicated that putative targets of Radix Salviae mostly participated in various pathways associated with angiogenesis, protein metabolism, inflammatory response, apoptosis, and cell proliferation. The putative targets of Radix Salviae (vascular endothelial growth factor, matrix metalloproteinases, plasminogen, insulin-like growth factor-1, and cyclooxygenase-2) were recognized as active factors involved in the main biological functions of treatment, which implied that these were involved in the underlying mechanisms of Radix Salviae on diabetic retinopathy.

Conclusions: Radix Salviae could alleviate diabetic retinopathy via the molecular mechanisms predicted by network pharmacology. This research demonstrates that the network pharmacology approach can be an effective tool to reveal the mechanisms of traditional Chinese medicine from a holistic perspective.

Keywords: Radix Salviae, Network pharmacology, Diabetic retinopathy

*Correspondence: pcl2013@sina.cn; 694397644@qq.com; tongxiaolin66@sina.com

${ }^{\dagger}$ Chun-Li Piao, Jin-Li Luo and De Jin are equal first authors ${ }^{1}$ Institution of Shenzhen Hospital, Guangzhou University of Chinese Medicine (Futian), Shenzhen 518000, Guangdong, China ${ }^{2}$ Institution of Guang'anmen Hospital, China Academy of Chinese Medical Science, Beijing 100000, China

\begin{abstract}
Introduction
Diabetic retinopathy (DR), which results from chronic high blood glucose levels, is one of the most common and serious complications of diabetes mellitus, which is the main cause of adult-acquired blindness [1]. A recent meta-analysis of 243 population-based studies shows that globally, in 2010, out of 32.4 million blind and 191 million visually impaired people, 0.8 million were blind
\end{abstract}


and 3.7 million were visually impaired because of DR [2]. Another meta-analysis, based on 288 studies, concluded that 2.6 million people were visually impaired (blindness and moderate to severe vision impairment) from DR in 2015 and this is estimated to rise to 3.2 million in 2020 [3]. Furthermore, DR leads to a poor quality of life and an increased risk of other diabetes complications and mortality, which brings severe social burden $[4,5]$. Currently, DR is mainly treated by laser photocoagulation of the retina, anti-vascular endothelial growth factor (VEGF) drug therapy, hormone therapy, and surgical treatment. However, these treatments might contribute to certain adverse reactions, such as the increase of angiogenesis, rise intraocular pressure, and retinal hemorrhage $[6,7]$ and the effect of single or combined treatment is limited. Therefore, it is urgent to discover potential therapeutic targets and develop new therapeutic strategies for the treatment of DR.

Traditional Chinese Medicine (TCM) is a comprehensive medicinal system that is characterized by its satisfying therapeutic effects and minor side effects. TCM is widely used in Asian countries, especially China [8]. TCM is characterized by multiple ingredients that have a variety of advantages, such as synergy, reduction of side effects, and improvement of adaptive resistance [9]. TCM network pharmacology not only identifies and optimizes multiple target interventions by modeling signaling pathways and specific processes [10], but also measures the efficacy of drugs, especially multi-target drugs [11]. Recently, TCM network pharmacology has been widely applied to the exploration of complex diseases, such as cancer, renal injury, and heart failure [12-14]. Based on the theory of traditional Chinese herbal medical science, TCM can offer a treatment for the prevention and treatment of DR in a systematic way. Radix Salviae is a species of Labiatae that is distributed throughout the country. As a traditional medicinal plant, it has satisfactory drug efficacy for the alleviation of DR, which indicates the existence of certain pharmacological components in Radix Salviae [15]. We discovered, in clinical practice, that Radix Salviae can effectively relieve the clinical symptoms of DR, such as local visual field defects, vision loss, and visual impairment [16]. However, the pharmacological mechanisms of Radix Salviae are still unknown.

With the rapid development of bioinformatics, systems biology, and poly-pharmacology, network pharmacology, based on the concept of "Disease-Gene-Target-Medicine", can explore the complex mechanisms of medicine on the human body [17]. This is in keeping with the holistic view of TCM and the mechanisms of TCM formulas are multiingredient, multi-pathway, and multi-target [18].

The aim of our study was to screen the related ingredients of Radix Salviae using multiple databases and acquire the potential targets by target fishing. Then, we aimed to screen the related targets of DR by consolidation of a multi-source database. Based on the matching results between Radix Salviae potential targets and DR targets, we aimed to build a protein-protein interaction (PPI) network to analyze the interactions among these targets and screen the hub targets based on topology. Moreover, using The Database for Annotation, Visualization and Integrated Discovery (DAVID) bioinformatics resources, we aimed to obtain the enrichment analysis of the Gene Ontology Biological Process (GO-BP) and Kyoto Encyclopedia of Genes and Genomes (KEGG). This study is necessary to investigate how Radix Salviae alleviates DR via the molecular mechanisms predicted by network pharmacology and how the network pharmacology approach can be an effective tool to reveal the mechanisms of TCM. The flowchart of the experimental procedures of our study is shown in Fig. 1.

\section{Methods \\ Data preparation \\ Chemical ingredients database building}

To collect the ingredients in Radix Salviae, we used the Traditional Chinese Medicine System Pharmacology Database [19] (TCMSP, http://lsp.nwu.edu.cn/tcmsp .php, 2019.8.11), a specialized pharmacological platform for TCM. Two hundred and two herbal ingredients were recorded in this process.

\section{Active ingredients screening Oral bioavailability $(O B)$ prediction}

$\mathrm{OB}$ is the percentage of an orally administered drug that reaches the systemic circulation. It is one of the most used pharmacokinetic properties in drug screening. In this process, the $\mathrm{OB}$ threshold was set as $30 \%$ and those ingredients with $\mathrm{OB} \geq 30 \%$ were selected as the active ingredients for the next step [20].

\section{Drug-likeness ( $D L$ ) evaluation}

$\mathrm{DL}$ is a molecular parameter that measures the absorption, distribution, metabolism, and excretion of drug molecules affected by their pharmacokinetics. By evaluating prospective "drug-like" compounds, DL optimizes the pharmacokinetic and pharmaceutical properties, such as chemical stability and solubility. The DL level of the compounds was set as 0.18 , as this is the selection criterion for "drug-like" compounds in traditional Chinese herbs [21]. In this study, those ingredients with $\mathrm{DL} \geq 0.18$ were selected.

\section{Target fishing}

The active ingredients of drugs play a role in related biological functions via targets. Our study located targets 


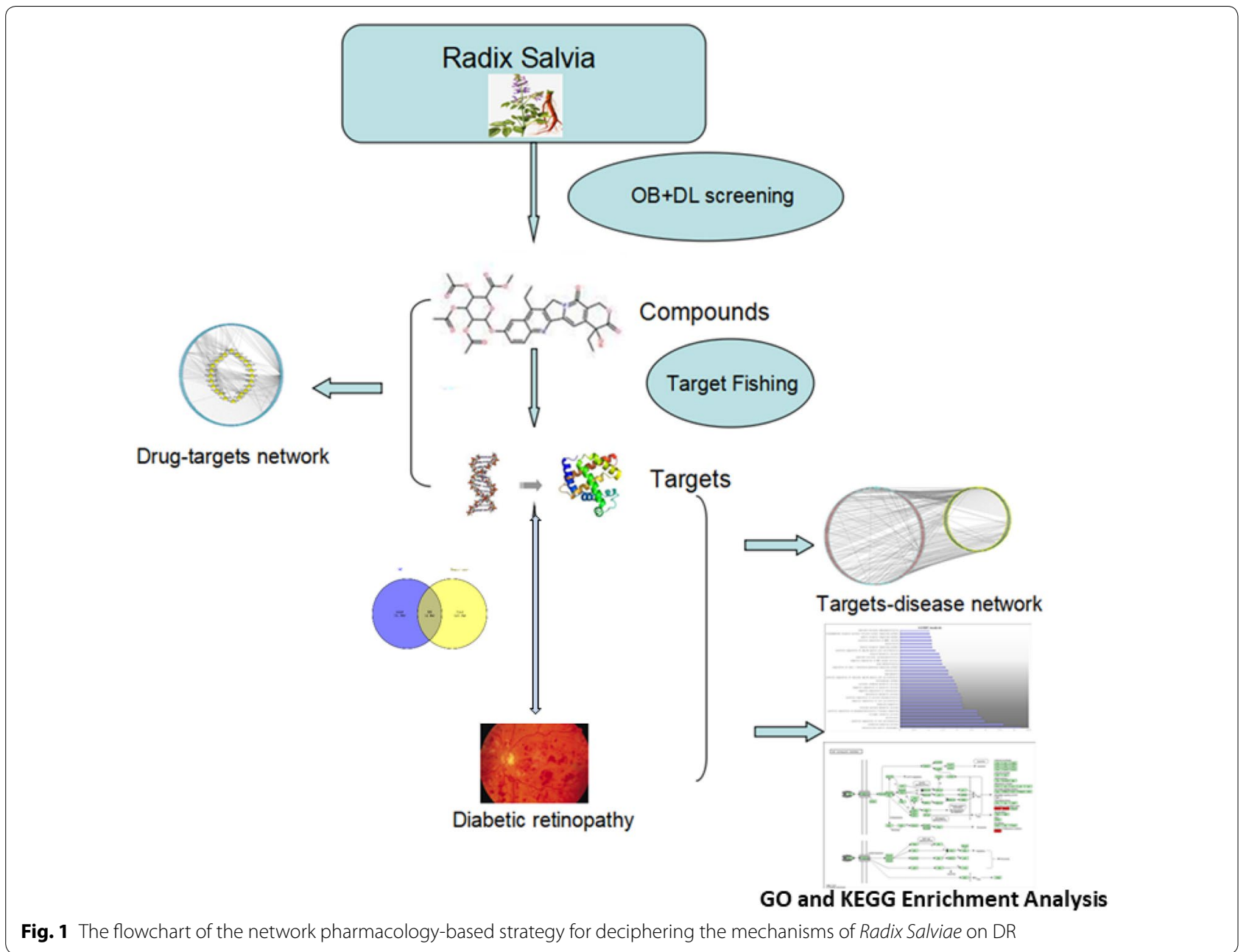

by target fishing based on the candidate ingredients. Retrieving the small molecular structure information of the active ingredients in Radix Salviae on the PubChem database (https://pubchem.ncbi.nlm.nih.gov/), we fished targets with a screening online tool called the Swiss Target Prediction webserver [22] (http://www.swisstarge tprediction.ch/index.php).

\section{Disease targets database building}

We collected DR targets from four source databases. The databases used in our study were: the DrugBank database (http://www.drugbank.ca/, version 4.3, 2019.8.11), Online Mendelian Inheritance in Man (OMIM) database [23] (http://www.omim.org/, 2019.8.11), DisGeNET v6 database [24] (http://www.disgenet.org/, version 6.0, 2019.8.11), and Genetic Association Database [25] (https ://www.geneticassociationdb.nih.gov/, 2019.8.11).

Finally, we matched the prediction of the targets of Radix Salviae active ingredients and the retrieval of the related targets of DR and chose the overlapping targets as the related targets of Radix Salviae for the treatment of DR. The targets were then processed by String [26] (https ://string-db.org/, 2019.8.13) to draw the data of PPI.

\section{Network construction Network construction method}

(1) Compound-target network (C-T network); (2) Radix Salviae target-DR target interactional network (T-T network); (3) Target-pathway network (T-P network). The pathway information of targets was screened from the result of KEGG pathway enrichment. Cytoscape3.6.0 (http://www.cytoscape.org/, 2019.8.14), an open-source software platform for visualizing complex networks and integrating these with any type of attribute data, helped build visualized network graphs [27].

\section{Network topological feature set definition}

We selected two parameters to evaluate the topological features of every node in the interaction network. "Degree" is defined as the number of links to a node, 
which reflects the frequency of interaction between a node and other nodes [28]. "Closeness Centrality" measures the mean distance from one node to another. A geodesic path is the shortest path through a network between nodes [29]. The more important the output of a node, the higher the value of this node in the network. Therefore, the levels of the two parameters represent the topological importance of the nodes in the network.

\section{Enrichment analysis}

We used DAVID (https://david.ncifcrf.gov/, v6.8,2019.8.14) [30] for GO enrichment analysis and KEGG (http://www.kegg.jp/, 2019.8.14) [31] for pathway enrichment analysis.

\section{Results}

\section{Active compounds in Radix Salviae}

Retrieved from TCMSP, there were 202 related components in the whole formula in total. According to the active ingredient screening thresholds of $\mathrm{OB} \geq 30 \%$ and $\mathrm{DL} \geq 0.18,65$ active ingredients were selected.

\section{Target prediction and analysis}

We conducted target fishing on the 65 active ingredients based on chemical similarity, obtaining 287 related targets. The 65 active compounds that were obtained are listed in Table 1.

The target data on DR from OMIM, Drugbank, GAD, and DisGeNet was integrated. Eighteen targets that matched the related targets of Radix Salviae on DR were collected as related targets for the effect of Radix Salviae on DR (Figs. 2 and 3).

In the String database, the PPI network of the 18 targets was established. The details are shown in Fig. 4. There were 18 nodes and 40 edges in total. The topological feature analysis of the PPI selected targets used median values to determine key targets and constructed the big hub nodes as the main targets that may cause the effect of Radix Salviae on DR, based on "degree" and "closeness certainty". The threshold values were degree $\geq 4.8$ and closeness $\geq 0.51$ and the results settled at 18 hub nodes and 40 edges. The details are shown in Fig. 5, which includes prostaglandin-endoperoxide synthase 2 (PTGS2) (degree =12), matrix metallopeptidase 9 (MMP9) (degree $=10)$, vascular endothelial growth factor receptor $2(\mathrm{KDR})$ (degree $=8)$, matrix metallopeptidase 2 (MMP2) (degree=7), plasminogen (PLG) (degree $=6)$, androgen receptor (degree $=5$ ), matrix metallopeptidase 3 (MMP3) (degree =5), and insulin-like growth factor $1(\mathrm{IGF}-1)$ receptor (degree $=5)$.

\section{GO biological process and KEGG pathway enrichment analysis}

DAVID v6.8 was used for enrichment analysis of the 18 targets. The screening threshold was $\mathrm{P}<0.01$ and $30 \mathrm{GO}$ items were retrieved. We selected 10 KEGG pathways for analysis.

\section{GO biological process enrichment analysis}

The 30 biological processes were mainly involved in angiogenesis, protein metabolism, inflammatory response, apoptosis, and cell proliferation. The details are shown in Fig. 6. The processes were, in the aspect of angiogenesis: angiogenesis (GO:0001525) and positive regulation of vascular smooth muscle cell proliferation (GO:1904707); in the aspect of protein metabolism: proteolysis (GO:0006508), collagen catabolic process (GO:0030574), cellular protein metabolic process (GO:0044267), negative regulation of fibrinolysis (GO:0051918), and fibrinolysis (GO:0042730); in the aspect of inflammatory response: regulation of type I interferon-mediated signaling pathway (GO:0060338), doxorubicin metabolic process (GO:0044598), and oxidation-reduction process (GO:0055114); in the aspect of apoptosis: negative regulation of apoptotic process (GO:0043066); and, in the aspect of cell proliferation: positive regulation of cell proliferation (GO:0008284), negative regulation of cell proliferation (GO:0008285), positive regulation of vascular smooth muscle cell proliferation (GO:1904707), and positive regulation of smooth muscle cell proliferation (GO:0048661). Based on these five main aspects, a complex multi-path synergetic effect may be the cause of the effect of Radix Salviae on DR.

\section{KEGG pathway enrichment analysis}

We conducted KEGG pathway enrichment analysis on 18 targets and screened 10 signaling pathways: pentose and glucuronate interconversions (hsa00040), glycerol-lipid metabolism (hsa00561), arachidonic acid metabolism (hsa00590), adherens junction (hsa04520), tumor necrosis factor signaling pathway (hsa04668), serotonergic synapse (hsa04726), linoleic acid metabolism (hsa00591), galactose metabolism (hsa00052), and fructose and mannose metabolism (hsa00051), and bladder cancer (hsa05219). The details are shown in Fig. 7.

\section{Discussion}

We discovered that Radix Salviae significantly alleviated the symptoms of DR and believe that its impact may be related to its potential function. Hence, we explored the potential mechanism by the network pharmacology approach. DR is one of the most common and serious microvascular complications in type 2 diabetes patients. 
Table 1 The list of 65 compounds of Radix Salviae and their OB and DL

\begin{tabular}{|c|c|c|c|}
\hline ID & Compound & OB & $\mathrm{DL}$ \\
\hline MOL001601 & 1,2,5,6-Tetrahydrotanshinone & 38.75 & 0.36 \\
\hline MOL001659 & Poriferasterol & 43.83 & 0.76 \\
\hline MOL001771 & Poriferast-5-en-3beta-ol & 36.91 & 0.75 \\
\hline MOL001942 & Isoimperatorin & 45.46 & 0.23 \\
\hline MOL002222 & Sugiol & 36.11 & 0.28 \\
\hline MOL002651 & Dehydrotanshinone II A & 43.76 & 0.4 \\
\hline MOL002776 & Baicalin & 40.12 & 0.75 \\
\hline MOL000569 & Digallate & 61.85 & 0.26 \\
\hline MOL000006 & Luteolin & 36.16 & 0.25 \\
\hline MOL006824 & a-Amyrin & 39.51 & 0.76 \\
\hline MOL007036 & 5,6-Dihydroxy-7-isopropyl-1,1-dimethyl-2,3-dihydrophenanthren-4-one & 33.77 & 0.29 \\
\hline MOL007041 & 2-Isopropyl-8-methylphenanthrene-3,4-dione & 40.86 & 0.23 \\
\hline MOL007045 & 3a-Hydroxytanshinolla & 44.93 & 0.44 \\
\hline MOL007048 & (E)-3-[2-(3,4-dihydroxyphenyl)-7-hydroxy-benzofuran-4-yl]acrylic acid & 48.24 & 0.31 \\
\hline MOL007049 & 4-Methylenemiltirone & 34.35 & 0.23 \\
\hline MOL007050 & 2-(4-Hydroxy-3-methoxyphenyl)-5-(3-hydroxypropyl)-7-methoxy-3-benzofurancarboxaldehyde & 62.78 & 0.4 \\
\hline MOL007051 & 6-o-Syringyl-8-o-acetyl shanzhiside methyl ester & 46.69 & 0.71 \\
\hline MOL007058 & Formyltanshinone & 73.44 & 0.42 \\
\hline MOL007059 & 3-Beta-hydroxymethyllenetanshiquinone & 32.16 & 0.41 \\
\hline MOL007061 & Methylenetanshinquinone & 37.07 & 0.36 \\
\hline MOL007063 & Przewalskin a & 37.11 & 0.65 \\
\hline MOL007064 & Przewalskin b & 110.32 & 0.44 \\
\hline MOL007068 & Przewaquinone B & 62.24 & 0.41 \\
\hline MOL007069 & Przewaquinone c & 55.74 & 0.4 \\
\hline MOL007070 & (6S,7R)-6,7-dihydroxy-1,6-dimethyl-8,9-dihydro-7H-naphtho[8,7-g]benzofuran-10,11-dione & 41.31 & 0.45 \\
\hline MOL007071 & Przewaquinone $f$ & 40.31 & 0.46 \\
\hline MOL007077 & Sclareol & 43.67 & 0.21 \\
\hline MOL007079 & Tanshinaldehyde & 52.47 & 0.45 \\
\hline MOL007081 & Danshenol B & 57.95 & 0.56 \\
\hline MOL007082 & Danshenol A & 56.97 & 0.52 \\
\hline MOL007085 & Salvilenone & 30.38 & 0.38 \\
\hline MOL007088 & Cryptotanshinone & 52.34 & 0.4 \\
\hline MOL007093 & Dan-shexinkum d & 38.88 & 0.55 \\
\hline MOL007094 & Danshenspiroketallactone & 50.43 & 0.31 \\
\hline MOL007098 & Deoxyneocryptotanshinone & 49.4 & 0.29 \\
\hline MOL007100 & Dihydrotanshinlactone & 38.68 & 0.32 \\
\hline MOL007101 & Dihydrotanshinone I & 45.04 & 0.36 \\
\hline MOL007105 & Epidanshenspiroketallactone & 68.27 & 0.31 \\
\hline MOL007107 & C09092 & 36.07 & 0.25 \\
\hline MOL007108 & Isocryptotanshi-none & 54.98 & 0.39 \\
\hline MOL007111 & Isotanshinone || & 49.92 & 0.4 \\
\hline MOL007115 & Manool & 45.04 & 0.2 \\
\hline MOL007118 & Microstegiol & 39.61 & 0.28 \\
\hline MOL007119 & Miltionone I & 49.68 & 0.32 \\
\hline MOL007120 & Miltionone II & 71.03 & 0.44 \\
\hline MOL007121 & Miltipolone & 36.56 & 0.37 \\
\hline MOL007122 & Miltirone & 38.76 & 0.25 \\
\hline MOL007123 & Miltirone II & 44.95 & 0.24 \\
\hline MOL007124 & Neocryptotanshinone ii & 39.46 & 0.23 \\
\hline
\end{tabular}


Table 1 (continued)

\begin{tabular}{|c|c|c|c|}
\hline ID & Compound & OB & DL \\
\hline MOL007125 & Neocryptotanshinone & 52.49 & 0.32 \\
\hline MOL007127 & 1-Methyl-8,9-dihydro-7H-naphtho[5,6-g]benzofuran-6,10,11-trione & 34.72 & 0.37 \\
\hline MOL007130 & Prolithospermic acid & 64.37 & 0.31 \\
\hline MOL007132 & (2R)-3-(3,4-dihydroxyphenyl)-2-[(Z)-3-(3,4-dihydroxyphenyl)acryloyl]oxy-propionic acid & 109.38 & 0.35 \\
\hline MOL007140 & (Z)-3-[2-[(E)-2-(3,4-dihydroxyphenyl)vinyl]-3,4-dihydroxy-phenyl]acrylic acid & 88.54 & 0.26 \\
\hline MOL007141 & Salvianolic acid g & 45.56 & 0.61 \\
\hline MOL007142 & Salvianolic acid j & 43.38 & 0.72 \\
\hline MOL007143 & Salvilenone I & 32.43 & 0.23 \\
\hline MOL007145 & Salviolone & 31.72 & 0.24 \\
\hline MOL007149 & NSC 122421 & 34.49 & 0.28 \\
\hline MOL007150 & (6S)-6-Hydroxy-1-methyl-6-methylol-8,9-dihydro-7H-naphtho[8,7-g]benzofuran-10,11-quinone & 75.39 & 0.46 \\
\hline MOL007151 & Tanshindiol B & 42.67 & 0.45 \\
\hline MOL007152 & Przewaquinone $\mathrm{E}$ & 42.85 & 0.45 \\
\hline MOL007154 & Tanshinone iia & 49.89 & 0.4 \\
\hline MOL007155 & (6S)-6-(hydroxymethyl)-1,6-dimethyl-8,9-dihydro-7H-naphtho[8,7-g]benzofuran-10,11-dione & 65.26 & 0.45 \\
\hline MOL007156 & Tanshinone VI & 45.64 & 0.3 \\
\hline
\end{tabular}

The basic pathological changes of DR include the loss of perithelial cell selectivity, thickening of the basement membrane, formation of microangioma, proliferation of endothelial cells, and neovascularization [32].

\section{Angiogenesis}

\section{We discovered that the anti-angiogenesis effect of Radix} Salviae may be a factor in its treatment of DR

During hyperglycemia, the activity of MMP9 is increased and the degradation of the basement membrane is accelerated, which degrades the cell matrix in the basement membrane, loosens the cell structure, and provides nutrients and growth space for the formation of new blood vessels [33]. KDR modulates angiogenic responses such as endothelial cell migration and proliferation. VEGF acts through highaffinity receptors and some consist of KDR [34].

VEGF is of major importance in proliferative DR [35] and can induce vascular abnormalities including vascular leakage and neovascularisation [36]. The VEGF and VEGFR system plays a major role in retinal neovacularization [37]. The inhibition of the binding of VEGF to its receptor can reduce neovacularization [38]. The role of VEGF in the pathogenesis of diabetic macular edema (DME) has been widely recognized. The intravitreal injection of anti-VEGF drugs has achieved good effects in the improvement of vision and reduction of macular edema and has become the initial therapy for DME [39]. Anti-VEGF treatment of proliferative DR may be superior to pan-retinal photocoagulation (PRP) and may delay or reduce the need for vitrectomy in vitreous hemorrhage cases when PRP is not possible [6]. Anti-VEGF therapeutics, including bevacizumab, ranibizumab, and aboxicept, are effective treatments for central macular edema [40].

PLG is a blood zymogen that is activated by proteolysis and converted to plasmin and angiostatin. Plasmin dissolves fibrin in blood clots and is an important protease in many other cellular processes, whereas angiostatin inhibits angiogenesis. Defects in PLG are likely a cause of thrombophilia. Tissue plasminogen activator (t-PA) and its inhibitor (PAI), participate in neovascularization, particularly in VEGF expression. Vascular tissue formation and new matrix component production is the basis of vascular proliferation. t-PA plays a major role in endothelial cell matrix degradation, which is an important precondition for endothelial cell proliferation and migration [41]. In addition, the expression of both t-PA and PAI is significantly correlated with VEGF expression [42].

IGF-1 stimulates growth, differentiation, and metabolism in a variety of cell types and plays a crucial role in both embryonic and postnatal growth. IGF-I is synthesized by the liver and acts on the tyrosine-kinase receptor (IGF-1R). IGF-1 expression is preserved in many tissues, including the retina [43]. Several retinal cell types, such as endothelial and retinal pigment epithelium cells, express both IGF-1 and its receptor. Injected IGF-1 induces retinal neovascularization and bloodretinal barrier breakdown in several in vitro studies [44].

\section{Apoptosis \\ We discovered that the inhibition of apoptosis induced by Radix Salviae may be a factor in its treatment of $D R$}

In the pathogenesis of DR, retinal microvascular cells (pericytes and endothelial cells) and other cells, including glial cells and neuronal cells, are lost selectively via 


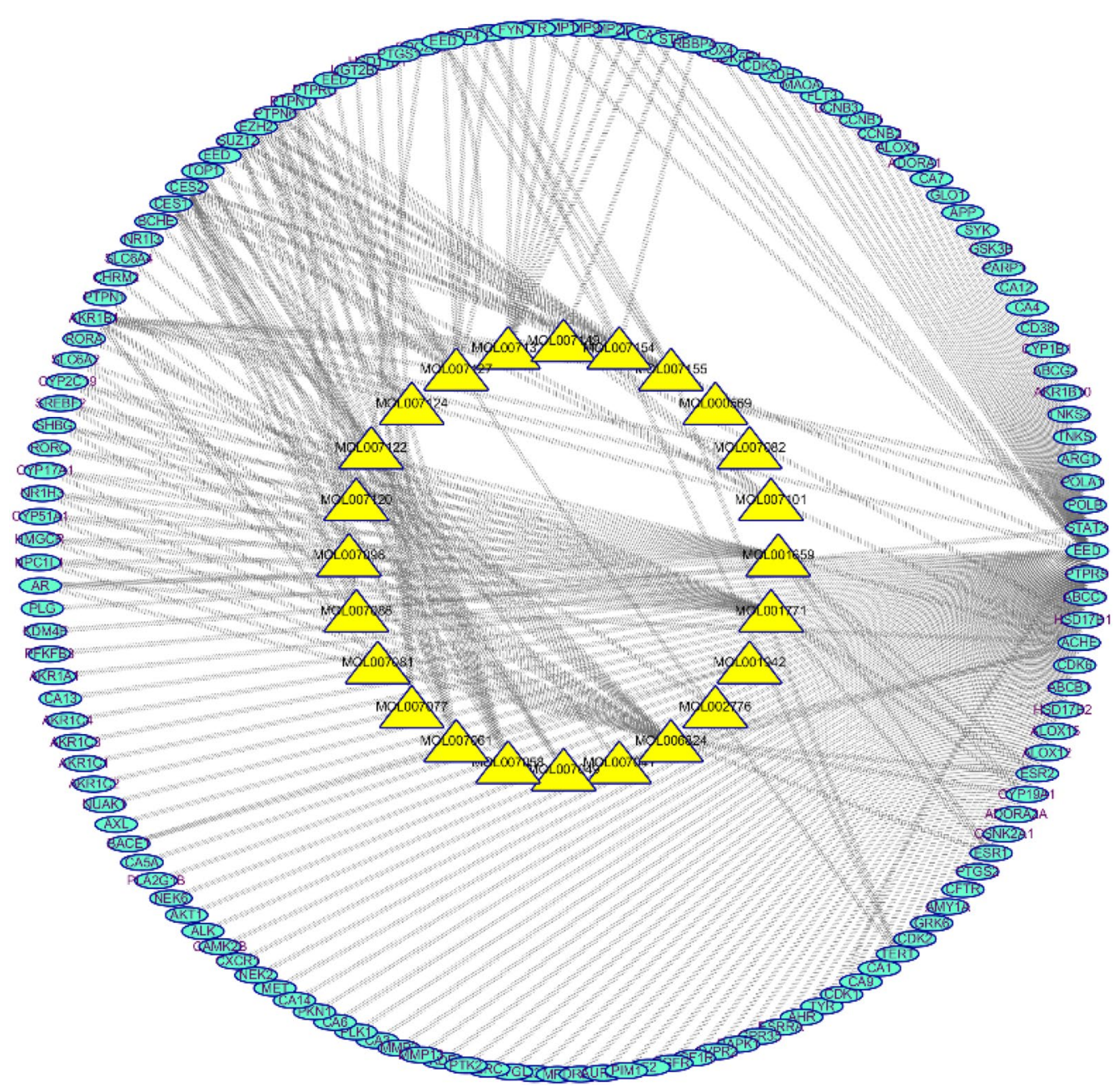

Fig. 2 The C-T network that consists of 24 nodes and 247 targets. Yellow and blue nodes denote the compounds and targets, respectively

apoptosis [45]. Apart from this, the accelerated loss of capillary cells may increase oxidative stress and inflammatory mediators [46]. In addition, the high level of manganese superoxide in the mitochondria has an important role in DR [47].

MMP, a member of the proteinase family, regulates major biological functions, including tissue repair and cell signaling. Among the MMPs, MMP2 is the most ubiquitous [48]. Activated MMP2 in the mitochondria results in the accelerated apoptosis of retinal capillary cells in diabetes, which damages the retinal mitochondria by modulating Hsp60 and connexin 43 and allows cytochrome c to leak out and activate apoptotic machinery [49].

\section{Inflammatory response}

We discovered that the induction of the inflammatory response by Radix Salviae may be a factor in its treatment of $D R$

Prostaglandin-endoperoxide synthase, also known as cyclooxygenase, is the key enzyme in prostaglandin biosynthesis and acts as both a dioxygenase and peroxidase. PTGS2, which is often called cyclooxygenase 2 (COX-2), is responsible for the prostanoid biosynthesis involved in inflammation and mitogenesis. In the plasma membrane, hyperglycemia activates aquaporin-1, which can sense osmolarity changes, and an "osmosignaling" pathway, which involves the transcription factor tonicity enhancer binding protein, which transmits the signal towards effector regulatory sites in the nuclei. This further promotes the expression of pro-inflammatory genes such as adhesion molecules and COX-2 [50]. 


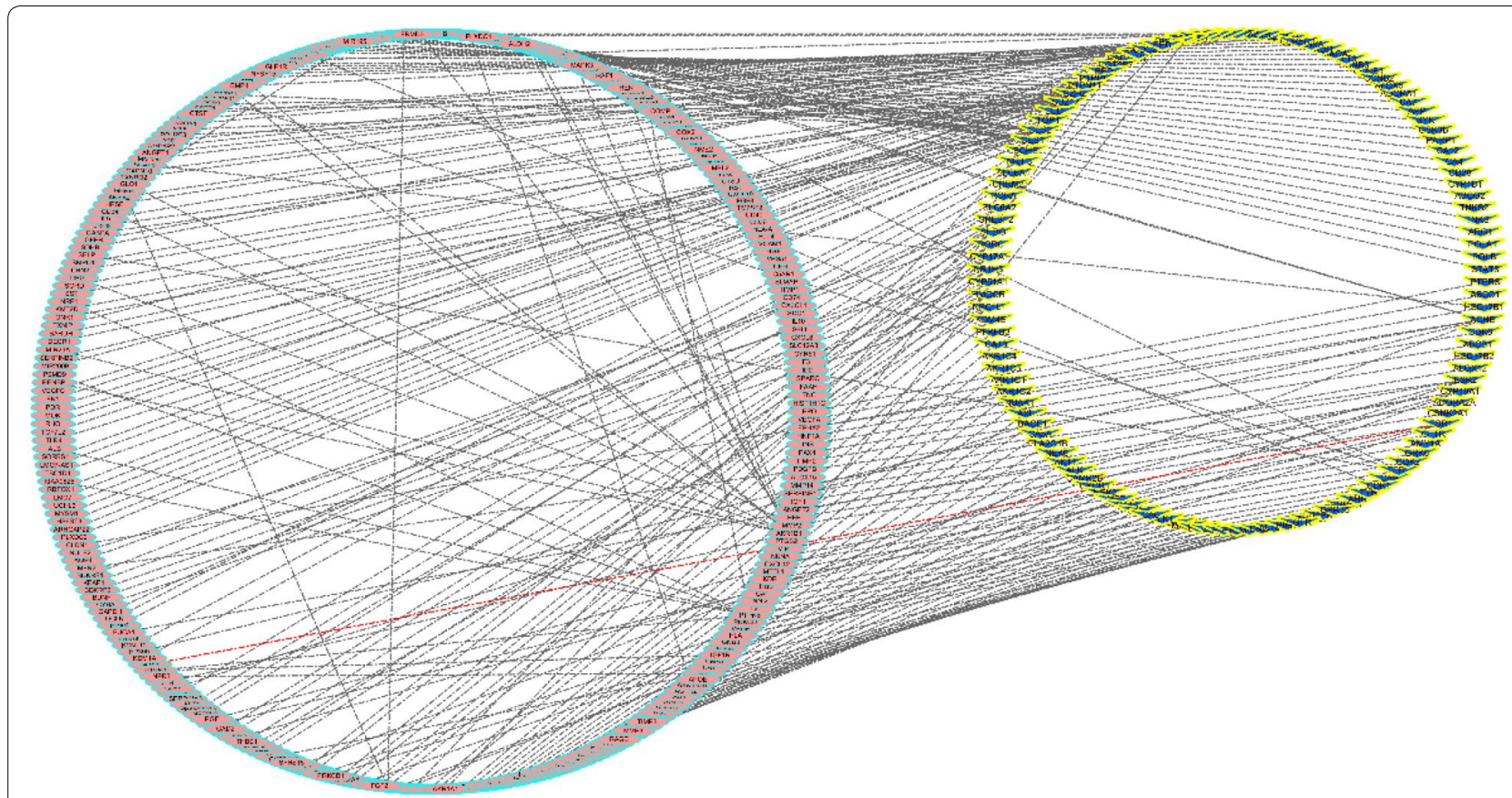

Fig. 3 The T-D network that consists of 287 nodes and 247 targets. Pink and yellow nodes denote the diseases and targets, respectively

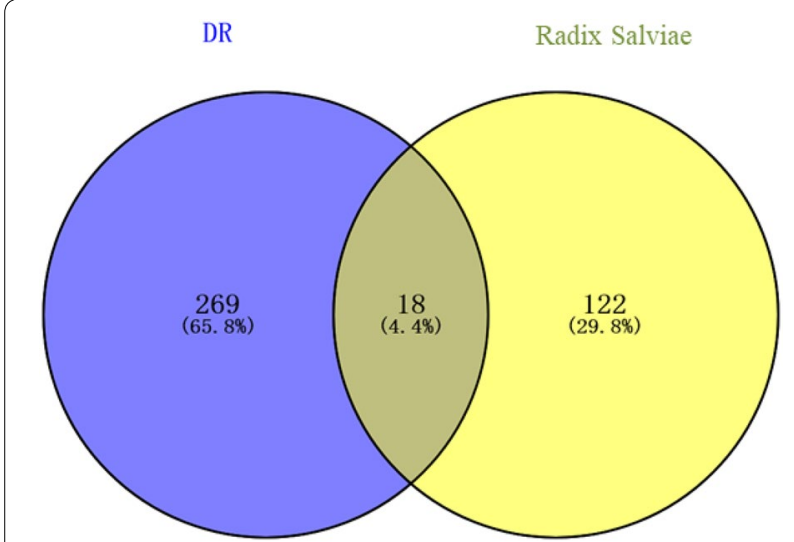

Fig. 4 The 18 matching targets of the related targets in Radix Salviae on DR. DR diabetic retinopathy

\section{Radix Salviae}

Radix Salviae is one of the most popular Chinese herbs and has been used for centuries for the management of cardiovascular and cerebrovascular diseases [51]. There are several studies that demonstrate that Radix Salviae can effectively reduce apoptosis, cell proliferation, and neovascularization. Liu et al. [52] believed that Danhong injection (DHI) could induce the expression of insulin receptor substrate 1, fibroblast growth factor 21, and peroxisome proliferator-activated receptor gamma

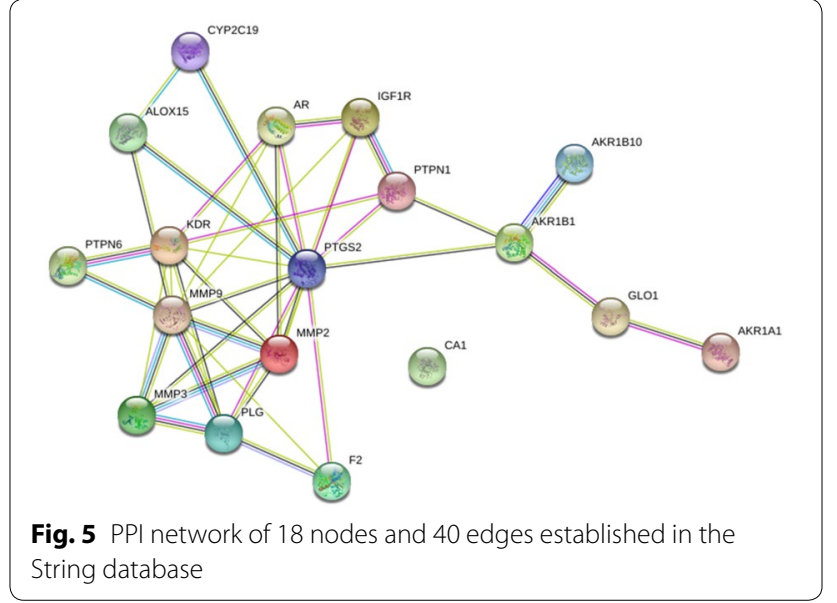

in the liver and peripheral tissues, which can increase insulin sensitivity. Furthermore, the induction of genes involved in lipolysis, fatty acid oxidation, and mitochondrial biogenesis suggests that DHI may enhance energy metabolism. Moreover, DHI inhibits CRISPR-associated gene 3, MMP2, and MMP9 expression and the formation of acellular capillaries in retinas; thus, DHI can prevent diabetes-induced apoptosis and protect retinas against diabetes-induced damage. Salvianolic acid B (Sal B), one of the major water-soluble compounds isolated from Radix Salviae, can inhibit high glucose-induced cell proliferation by releasing the cell from G1 phase arrest and 


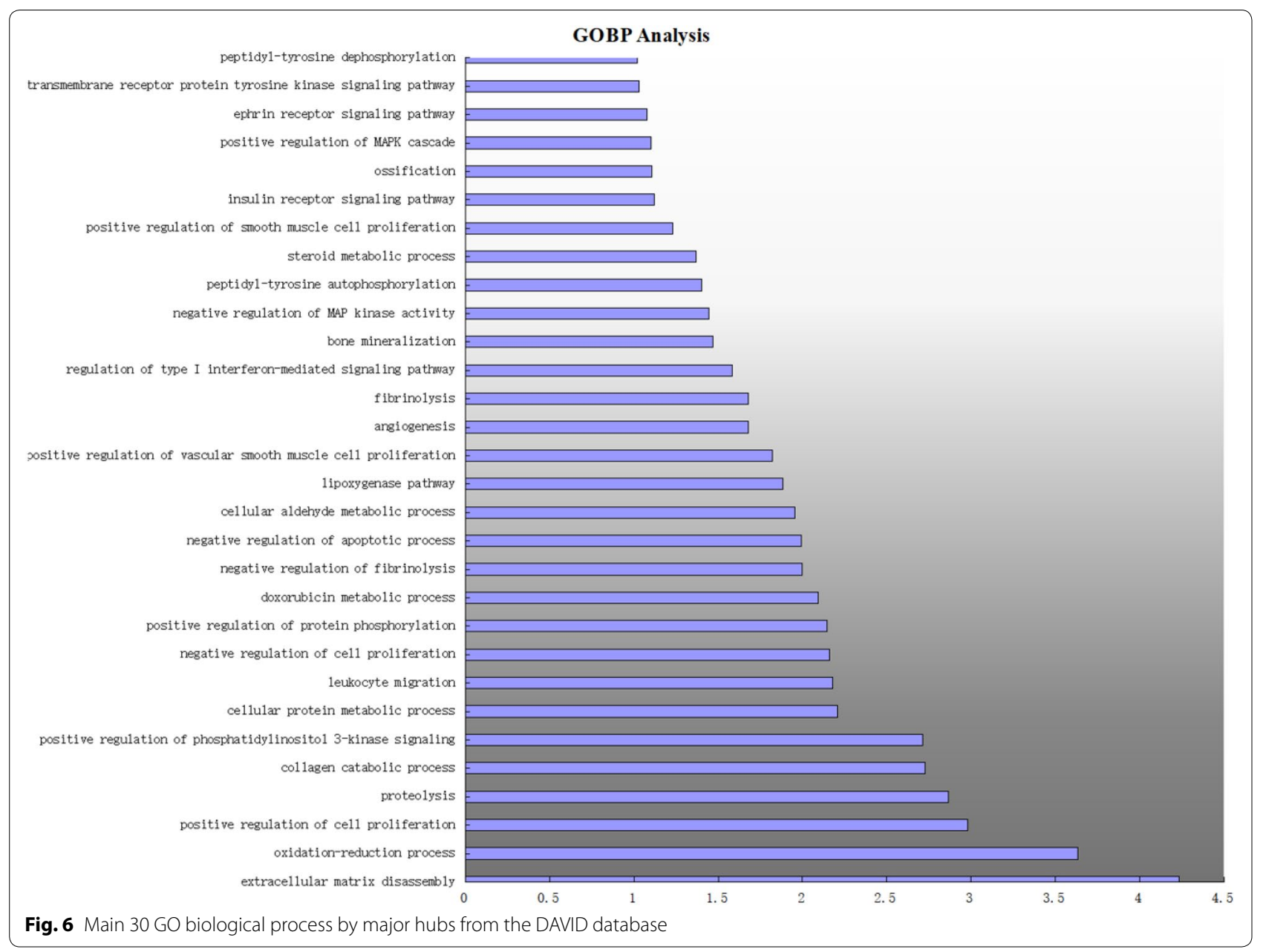

\section{KEGG Analysis}

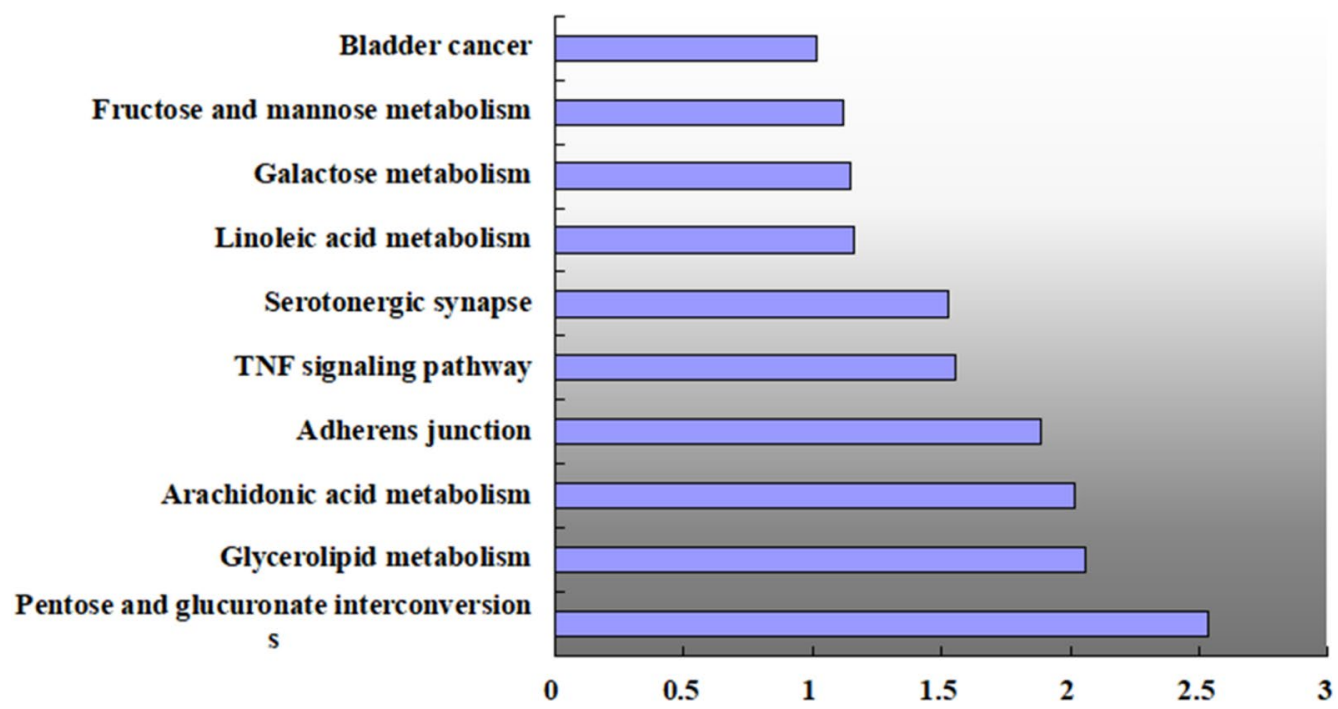

Fig. 7 The main 10 pathways enriched by major hubs from the DAVID database 
delaying $\mathrm{S}$ phase progression in human mesangial cells. Sal B can decrease the secretion of high glucose-induced MMP2 and MMP9, which is partly mediated by blocking NF- $\mathrm{kB}$ activation [53]. Sal A has a wide range of pharmacological effects, such as anti-inflammatory, antioxidant, and anti-fibrotic properties [51, 54, 55]. In addition, the Chinese herbal compound Radix Salviae is widely used in clinical practice. A recent meta-analysis shows that the herb Salvia miltiorrhiza (Radix Salviae dripping pill, CDDP) can protect endothelial diastolic function, vision, and visual acuity, improve microvascular structure, and improve retinal microcirculation. Therefore, Radix Salviae can safely and effectively delay the progression of DR and loss of vision, providing a new treatment for DR (Fig. 8).

However, there are some limitations in the use of network pharmacological methods to predict active ingredients and potential mechanisms. (i) The screened active ingredients may be inconsistent with the actual absorbed components in the blood of patients with DR; (ii) it may be difficult to distinguish the inhibitory effect from the target activation effect; (iii) the predicted results may be affected by possible deviation in highly studied pathways and functions; and (iv) the interaction relationships between the nodes in the network construction methods are still unclear. Therefore, further experimental verification of the potential active ingredients is needed to verify this theoretical prediction.

\section{Conclusions}

This study used a scientific approach to decipher the pharmacological mechanisms of Radix Salviae in the treatment of DR. We discovered that the effects may be associated with anti-angiogenesis, the inhibition of apoptosis, and the inflammatory response. Among these crucial biological functions, eight targets were identified as key active factors involved in the related pathways. This research suggests that Radix Salviae can alleviate DR via the molecular mechanisms predicted by network pharmacology and that the network pharmacology approach

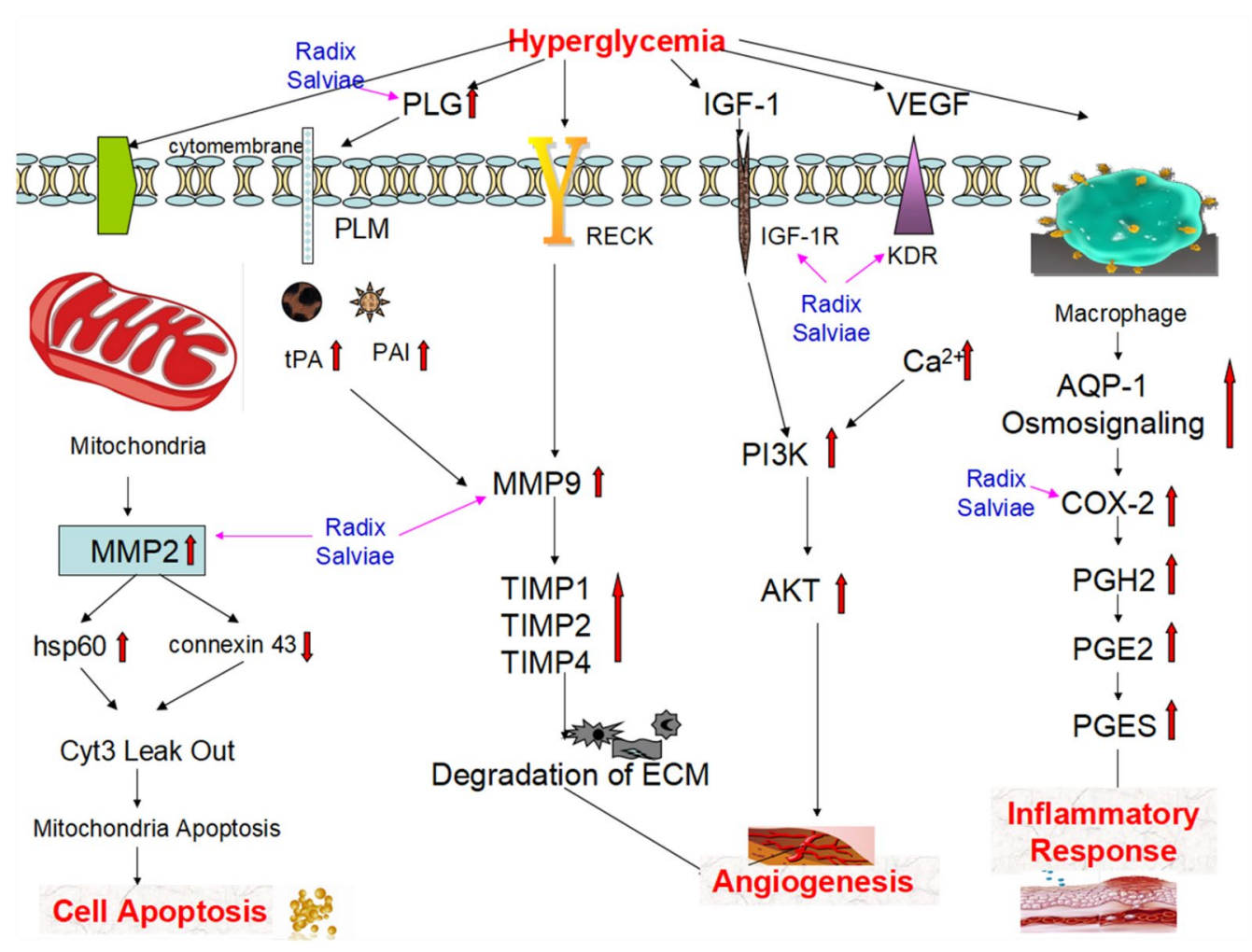

Fig. 8 Illustration of the crucial biological processes caused by putative targets and known therapeutic targets for DR. MMP2 matrix metallopeptidase 2, hsp60 heat shock protein 60, Cyt3 cytochrome 3, PLG plasminogen, PLM plasmin, IPA tissue plasminogen activator, PAI plasminogen activator inhibitor, RECK reversion-inducing cysteine-rich protein with Kazal motifs, MMP9 matrix metallopeptidase 9, TIMP 1 tissue inhibitor of metalloproteinases 1, TIMP 2 tissue inhibitor of metalloproteinases 2, TIMP 4 tissue inhibitor of metalloproteinases 4, ECM extracellular matrix, IGF-1 insulin-like growth factor-1, IGF-1R insulin-like growth factor-1 receptor, PI3K phosphatidylinositol 3-kinase, AKT serine-threonine kinase, VEGF vascular endothelial growth factor, KDR vascular endothelial growth factor receptor 2, AQP-1 aquaporin-1, COX-2 cyclooxygenase-2, PGH2 prostaglandin $\mathrm{H} 2$, PGE2 prostaglandin E2, PGES prostaglandin E2 synthase 
can be an effective tool to reveal the mechanisms of TCM. However, to improve the reliability of the results, further experimental experiments are needed to validate these results.

\begin{abstract}
Abbreviations
DR: diabetic retinopathy; VEGF: vascular endothelial growth factor; TCM: Traditional Chinese Medicine; OB: oral bioavailability; DL: drug-likeness; GO: Gene Ontology; KEGG: Kyoto Encyclopedia of Genes and Genomes; PPI: protein-protein interaction; DAVID: Database for Annotation, Visualization and Integrated Discovery; C-T: compound-target; T-T: target-target; T-P: targetpathway; PTGS2: prostaglandin-endoperoxide synthase 2; MMP3: matrix metalloprotease 3; MMP2: matrix metallopeptidase 2; PLG: plasminogen; PLM: plasmin; tPA: tissue plasminogen activator; PAl: plasminogen activator inhibitor; MMP9: matrix metallopeptidase 9; IGF-1: insulin-like growth factor-1; IGF-1R: insulin-like growth factor-1 receptor; KDR: vascular endothelial growth factor receptor 2; COX-2: cyclooxygenase-2; PRP: pan-retinal photocoagulation; DHI: Danhong injection.
\end{abstract}

\section{Acknowledgements}

Not applicable.

\section{Authors' contributions}

F-ML and X-LT conceived and designed the research methods. C-LP, J-LL and DJ collected the data. C-LP and DJ analyzed the data. CT and LW provided advice. J-LL wrote the paper. All authors read and approved the final manuscript.

\section{Funding}

This work was supported by the 2015 Traditional Chinese Medicine Scientific Research (Grant Number: 201507001-11) and The National Natural Science Foundation of China (Grant Number: 81973813).

\section{Availability of data and materials}

The data and materials generated or analyzed during this study are available from the corresponding author on reasonable request.

\section{Consent for publication}

The manuscript is approved by all authors for publication.

\section{Competing interests}

The authors declare that they have no competing interests.

Received: 19 October 2019 Accepted: 17 December 2019

Published online: 30 December 2019

\section{References}

1. American Academy of Ophthalmology Retina/Vitreous Panel. Preferred Practice Pattern ${ }^{\circledR}$ Guidelines. Diabetic retinopathy. San Francisco, CA: American Academy of Ophthalmology; 2017. http://www.aao.org/ppp. Accessed 15 Aug 2019.

2. Leasher JL, Bourne RR, Flaxman SR, et al. Erratum. Global estimates on the number of people blind or visually impaired by diabetic retinopathy: a meta-analysis from 1990 to 2010. Diabetes Care. 2016;39:1643-9. Diabetes Care. 2016;39(11): 2096.

3. Flaxman SR, Bourne RRA, Resnikoff S, et al. Global causes of blindness and distance vision impairment 1990-2020: a systematic review and metaanalysis. Lancet Glob Health. 2017;5(12):e1221-34.

4. Rees G, Xie J, Fenwick EK, et al. Association between diabetes-related eye complications and symptoms of anxiety and depression. JAMA Ophthalmol. 2016;134(9):1007-14

5. Kramer CK, Rodrigues TC, Canani LH, Gross JL, Azevedo MJ. Diabetic retinopathy predicts all-cause mortality and cardiovascular events in both type 1 and 2 diabetes: meta-analysis of observational studies. Diabetes Care. 2011;34(5):1238-44.

6. Zhao Y, Singh RP. The role of anti-vascular endothelial growth factor (antiVEGF) in the management of proliferative diabetic retinopathy. Drugs Context. 2018;7:212532.

7. Whitcup SM, Cidlowski JA, Csaky KG, Ambati J. Pharmacology of corticosteroids for diabetic macular edema. Invest Ophthalmol Vis Sci. 2018;59(1):1-12.

8. Li S, Xutian S. New development in Traditional Chinese Medicine: symbolism-digit therapy as a special naturopathic treatment. Am J Chin Med. 2016:44(7):1311-23.

9. Li S, Zhang B, Zhang N. Network target for screening synergistic drug combinations with application to Traditional Chinese Medicine. BMC Syst Biol. 2011;5(Suppl 1):S10.

10. Fitzgerald JB, Schoeberl B, Nielsen UB, Sorger PK. Systems biology and combination therapy in the quest for clinical efficacy. Nat Chem Biol. 2006;2(9):458-66.

11. Csermely P, Agoston V, Pongor S. The efficiency of multi-target drugs: the network approach might help drug design. Trends Pharmacol Sci. 2005;26(4):178-82.

12. Zheng J, Wu M, Wang $\mathrm{H}$, et al. Network pharmacology to unveil the biological basis of health-strengthening herbal medicine in cancer treatment. Cancers. 2018;10(11):E461.

13. Xu L, Zhang Y, Zhang P, et al. Integrated metabolomics and network pharmacology strategy-driven active traditional chinese medicine ingredients discovery for the alleviation of cisplatin nephrotoxicity. Chem Res Toxicol. 2019;32:2411-21.

14. Liu J, Li Y, Zhang Y, et al. A Network Pharmacology Approach to Explore the Mechanisms of Qishen Granules in Heart Failure. Med Sci Monit Int Med J Exp Clin Res. 2019;25:7735-45.

15. Dong Z, Tao X, Fu X, Wang H, Wang D, Zhang T. Protective effects of Purendan superfine powder on retinal neuron apoptosis in a rat model of type 2 diabetes mellitus. Neural Regen Res. 2012;7(3):202-6.

16. Lian F, Wu L, Tian J, et al. The effectiveness and safety of a danshen-containing Chinese herbal medicine for diabetic retinopathy: a randomized, double-blind, placebo-controlled multicenter clinical trial. J Ethnopharmacol. 2015;164:71-7.

17. Hopkins AL. Network pharmacology: the next paradigm in drug discovery. Nat Chem Biol. 2008;4(11):682-90.

18. Zhang Y, Bai M, Zhang B, et al. Uncovering pharmacological mechanisms of Wu-tou decoction acting on rheumatoid arthritis through systems approaches: drug-target prediction, network analysis and experimental validation. Sci Rep. 2015;5:9463.

19. Ru J, Li P, Wang J, et al. TCMSP: a database of systems pharmacology for drug discovery from herbal medicines. J Cheminform. 2014;6:13.

20. Xu X, Zhang W, Huang C, et al. A novel chemometric method for the prediction of human oral bioavailability. Int J Mol Sci. 2012;13(6):6964-82.

21. Tao $W, X u X$, Wang $X$, et al. Network pharmacology-based prediction of the active ingredients and potential targets of Chinese herbal Radix Curcumae formula for application to cardiovascular disease. J Ethnopharmacol. 2013:145(1):1-10.

22. Gfeller D, Michielin O, Zoete V. Shaping the interaction landscape of bioactive molecules. Bioinformatics. 2013;29(23):3073-9.

23. Amberger JS, Hamosh A. Searching online mendelian inheritance in man (OMIM): a knowledgebase of human genes and genetic phenotypes. Curr Protoc Bioinformatics. 2017;58:1.2.1-2.12.

24. Pinero J, Bravo A, Queralt-Rosinach N, et al. DisGeNET: a comprehensive platform integrating information on human disease-associated genes and variants. Nucleic Acids Res. 2017;45(D1):D833-d839.

25. Wang H, Liu X, Tao Y, et al. Automatic human-like mining and constructing reliable genetic association database with deep reinforcement learning. Pac Symp Biocomput. 2019;24:112-23.

26. Szklarczyk D, Morris JH, Cook H, et al. The STRING database in 2017: quality-controlled protein-protein association networks, made broadly accessible. Nucleic Acids Res. 2017;45(D1):D362-d368.

27. Shannon P, Markiel A, Ozier O, et al. Cytoscape: a software environment for integrated models of biomolecular interaction networks. Genome Res. 2003;13(11):2498-504. 
28. Missiuro PV, Liu K, Zou L, et al. Information flow analysis of interactome networks. PLoS Comput Biol. 2009;5(4):e1000350.

29. Tang Y, Li M, Wang J, Pan Y, Wu FX. CytoNCA: a cytoscape plugin for centrality analysis and evaluation of protein interaction networks. Biosystems. 2015;127:67-72.

30. da Huang W, Sherman BT, Lempicki RA. Systematic and integrative analysis of large gene lists using DAVID bioinformatics resources. Nat Protoc. 2009;4(1):44-57.

31. Chen L, Zhang YH, Wang S, Zhang Y, Huang T, Cai YD. Prediction and analysis of essential genes using the enrichments of gene ontology and KEGG pathways. PLoS ONE. 2017;12(9):e0184129.

32. Lechner J, O'Leary OE, Stitt AW. The pathology associated with diabetic retinopathy. Vision Res. 2017;139:7-14.

33. Herszenyi L, Hritz I, Pregun I, et al. Alterations of glutathione S-transferase and matrix metalloproteinase-9 expressions are early events in esophageal carcinogenesis. World J Gastroenterol. 2007;13(5):676-82.

34. Li F, Huang J, Ji D, et al. Azithromycin effectively inhibits tumor angiogenesis by suppressing vascular endothelial growth factor receptor 2-mediated signaling pathways in lung cancer. Oncol Lett. 2017;14(1):89-96.

35. Thomas KA. Vascular endothelial growth factor, a potent and selective angiogenic agent. J Biol Chem. 1996;271(2):603-6.

36. Weis SM, Cheresh DA. Pathophysiological consequences of VEGF-induced vascular permeability. Nature. 2005;437(7058):497-504.

37. Adams RH, Alitalo K. Molecular regulation of angiogenesis and lymphangiogenesis. Nat Rev Mol Cell Biol. 2007;8(6):464-78.

38. Olmos LC, Sayed MS, Moraczewski AL, et al. Long-term outcomes of neovascular glaucoma treated with and without intravitreal bevacizumab. Eye (Lond). 2016;30(3):463-72

39. Cai S, Yang Q, Li X, Zhang Y. The efficacy and safety of aflibercept and conbercept in diabetic macular edema. Drug Des Devel Ther. 2018;12:3471-83.

40. Wells JA, Glassman AR, Ayala AR, et al. Aflibercept, bevacizumab, or ranibizumab for diabetic macular edema. N Engl J Med. 2015;372(13):1193-203.

41. Singh R, Ramasamy K, Abraham C, Gupta V, Gupta A. Diabetic retinopathy: an update. Indian J Ophthalmol. 2008;56(3):178-88

42. Wu SL, Zhan DM, Xi SH, He XL. Roles of tissue plasminogen activator and its inhibitor in proliferative diabetic retinopathy. Int J Ophthalmol. 2014;7(5):764-7.

43. Ruiz de Almodovar C, Luttun A, Carmeliet P. An SDF-1 trap for myeloid cells stimulates angiogenesis. Cell. 2006;124(1):18-21.
44. Le Roith D, Bondy C, Yakar S, Liu JL, Butler A. The somatomedin hypothesis: 2001. Endocr Rev. 2001;22(1):53-74.

45. Poulaki , Joussen AM, Mitsiades N, Mitsiades CS, lliaki EF, Adamis AP. Insulin-like growth factor-I plays a pathogenetic role in diabetic retinopathy. Am J Pathol. 2004;165(2):457-69.

46. Roy $S, A m i n ~ S$, Roy S. Retinal fibrosis in diabetic retinopathy. Exp Eye Res. 2016;142:71-5.

47. Shin YI, Nam KY, Lee SE, et al. Peripapillary microvasculature in patients with diabetes mellitus: an optical coherence tomography angiography study. Sci Rep. 2019;9(1):15814.

48. Barber AJ, Lieth E, Khin SA, Antonetti DA, Buchanan AG, Gardner TW. Neural apoptosis in the retina during experimental and human diabetes. Early onset and effect of insulin. J Clin Invest. 1998;102(4):783-91.

49. Mohammad G, Kowluru RA. Novel role of mitochondrial matrix metalloproteinase-2 in the development of diabetic retinopathy. Invest Ophthalmol Vis Sci. 2011;52(6):3832-41.

50. Madonna R, Giovannelli G, Confalone P, Renna FV, Geng YJ, De Caterina R. High glucose-induced hyperosmolarity contributes to COX-2 expression and angiogenesis: implications for diabetic retinopathy. Cardiovasc Diabetol. 2016;15:18.

51. Tang MK, Ren DC, Zhang JT, Du GH. Effect of salvianolic acids from Radix Salviae miltiorrhizae on regional cerebral blood flow and platelet aggregation in rats. Phytomedicine. 2002;9(5):405-9.

52. Liu M, Pan Q, Chen Y, et al. Administration of Danhong Injection to diabetic $\mathrm{db} / \mathrm{db}$ mice inhibits the development of diabetic retinopathy and nephropathy. Sci Rep. 2015;5:11219.

53. Luo $P, \operatorname{Tan} Z$, Zhang Z, Li H, Mo Z. Inhibitory effects of salvianolic acid B on the high glucose-induced mesangial proliferation via NF-kappaBdependent pathway. Biol Pharm Bull. 2008;31(7):1381-6.

54. Yang LL, Li DY, Zhang YB, Zhu MY, Chen D, Xu TD. Salvianolic acid A inhibits angiotensin II-induced proliferation of human umbilical vein endothelial cells by attenuating the production of ROS. Acta Pharmacol Sin. 2012;33(1):41-8.

55. Li HY, Li Y, Yan CH, Li LN, Chen XG. Inhibition of tumor growth by S-3-1, a synthetic intermediate of salvianolic acid A. J Asian Nat Prod Res. 2002;4(4):271-80.

\section{Publisher's Note}

Springer Nature remains neutral with regard to jurisdictional claims in published maps and institutional affiliations.
Ready to submit your research? Choose BMC and benefit from:

- fast, convenient online submission

- thorough peer review by experienced researchers in your field

- rapid publication on acceptance

- support for research data, including large and complex data types

- gold Open Access which fosters wider collaboration and increased citations

- maximum visibility for your research: over $100 \mathrm{M}$ website views per year

At BMC, research is always in progress.

Learn more biomedcentral.com/submissions 